\title{
Sensitivity of Hawking radiation to superluminal dispersion relations
}

\author{
C. Barceló, ${ }^{1}$ L. J. Garay, ${ }^{2,3}$ and G. Jannes ${ }^{1,3}$ \\ ${ }^{1}$ Instituto de Astrofísica de Andalucía, CSIC, Camino Bajo de Huétor 50, 18008 Granada, Spain \\ ${ }^{2}$ Departamento de Física Teórica II, Universidad Complutense de Madrid, 28040 Madrid, Spain \\ ${ }^{3}$ Instituto de Estructura de la Materia, CSIC, Serrano 121, 28006 Madrid, Spain
}

(Received 25 July 2008; published 22 January 2009)

\begin{abstract}
We analyze the Hawking radiation process due to collapsing configurations in the presence of superluminal modifications of the dispersion relation. With such superluminal dispersion relations, the horizon effectively becomes a frequency-dependent concept. In particular, at every moment of the collapse, there is a critical frequency above which no horizon is experienced. We show that, as a consequence, the late-time radiation suffers strong modifications, both quantitative and qualitative, compared to the standard Hawking picture. Concretely, we show that the radiation spectrum becomes dependent on the measuring time, on the surface gravities associated with different frequencies, and on the critical frequency. Even if the critical frequency is well above the Planck scale, important modifications still show up.
\end{abstract}

DOI: 10.1103/PhysRevD.79.024016

PACS numbers: 04.70.Dy, 04.60.Bc, 04.62.+v

\section{INTRODUCTION}

Phenomenological approaches to quantum gravity have recently started to develop in parallel with the more traditional attempts to construct such a theory from first principles. In particular, increasing attention has focused on the consideration that maybe Lorentz invariance is not a fundamental law, but an effective low-energy symmetry which is broken at high energies (see, e.g., [1] for a general introduction). Conceptually speaking, in quantum gravity theories from first principles, it is not really clear whether Lorentz invariance is fundamental or effective, and in the latter case, how its breaking scale is related to the Planck scale. For example, while many string theory scenarios axiomatically incorporate Lorentz invariance, it has been argued that in certain situations, violations of Lorentz invariance may occur in a way consistent with world-sheet conformal invariance [2], thus leading to acceptable string theory backgrounds. In the context of loop quantum gravity, in [3] it has been argued that quantum effects should modify the relativistic dispersion relations, although the issue seems far from settled (see [4] for some general remarks). In scenarios of emergent gravity based on condensed matter analogies [5,6], the situation is clearer: Lorentz invariance is a low-energy effective symmetry, and so it is expected to break at some scale, although not necessarily related to (and therefore possibly much higher than) the Planck scale [7].

A simple way of modelling a wide range of Lorentz violating effects (and a quite natural one in the case of condensed matter analogies) consists in modifying the dispersion relations at high energy [8]. This modification can be subluminal or superluminal, depending on whether high-frequency modes move slower or faster than low- energy ones. We should mention that there also exist ways of modifying the dispersion relations, for example, by introducing a minimum length or maximum energy, without violating Lorentz invariance [9]. Here, however, we will be interested in modifications that explicitly break Lorentz invariance. From extrapolation of current experiments, we know that there exist stringent bounds on the most commonly expected types of Lorentz violations at the Planck scale (see, e.g., [1] and references therein). Nevertheless, even violations at much higher energy scales might still significantly affect black hole physics.

In this paper we will investigate the effects that a superluminal modification of the dispersion relation would have on the Hawking radiation produced by collapsing configurations. Hawking's original prediction that black holes radiate thermally $[10,11]$, rested on the implicit assumption that the low-energy laws of physics, and, in particular, Lorentz invariance, are preserved up to arbitrarily large scales, much higher than the Planck energy. The question of the robustness of Hawking's prediction to modifications of the trans-Planckian physics has been tackled principally by analyzing effective field theories such that at high energies a modification of the dispersion relation is incorporated (see, however, [12] for a different take on the problem). Historically, attention has mainly been given to subluminal dispersion relations. Important contributions such as [13-20], and more recently [21,22] seem to have settled the robustness of Hawking radiation with respect to subluminal modifications and truncation of the frequency spectrum, although this conclusion still rests on certain assumptions, usually related to the behavior of the fields near the horizon. In any case, it is important to remember that this only solves part of the so-called "trans-Planckian problem" with regard to Hawking radiation. Indeed, sub- 
luminal modifications gradually dampen the influence of ultrahigh frequencies, and so they do not explore arbitrarily large frequencies. So even assuming that it has been demonstrated that Hawking's result can be recovered without appeal to trans-Planckian frequencies, and that it is robust to subluminal modifications, the question remains whether it is also robust with respect to a (nondampened) modification of the trans-Planckian physics. Superluminal modifications differ conceptually from subluminal ones, in that they gradually magnify the influence of ultrahigh energies, and thereby offer an interesting test-case for the transPlanckian robustness of Hawking radiation.

An additional motivation for our study comes from a possible connection with experiment. It is well known (see, e.g., [23]) that Hawking radiation is not a purely gravitational effect, but a characteristic of quantum field theory in curved spacetimes with a horizon. Condensed matter systems such as Bose-Einstein condensates (BECs) can, under certain approximations, be described by a relativistic quantum field theory. It has also been shown, again under certain idealizing approximations, that (acoustic) black hole horizons in BECs are dynamically stable [24]. ${ }^{1}$ Therefore, BECs are expected to be good candidates for a possible experimental verification of Hawking radiation [26-34]. But since the physics of BECs automatically leads to a superluminal dispersion relation at high energies, the question is again which kind of modifications are to be expected in the laboratory realization of a BEC black hole with respect to the standard Hawking picture.

Of the above-mentioned works on the robustness of Hawking radiation, a few have also tried to address superluminal modifications [20-22]. Various problems make this case quite different from the subluminal one. These problems can be related to the fact that the horizon becomes frequency dependent when modifying the dispersion relations. This is also true in the subluminal case, though qualitatively in a very different way. With superluminal modifications, the horizon lies ever closer to the singularity for increasing frequencies, and asymptotically coincides with it. This causes the "apparent" interior of the black hole (the interior of the zero-frequency horizon) to be exposed to the outside world. Since it seems unreasonable to impose a condition arbitrarily close to the singularity as long as we do not have a solidly confirmed quantum theory of gravity, most of the approaches used for subluminal dispersion are invalid, or at least questionable, for super-

\footnotetext{
${ }^{1}$ Note, however, that this dynamical stability is quite fragile. For example, a minimal amount of backscattering in the interior of the black hole could be sufficient to create dynamical instabilities [24], which in a realistic laboratory experiment might completely obfuscate any quantum radiation related to an analogue Hawking process. This instability was first revealed in a mode analysis of a black hole-white hole configuration under a superluminal dispersion relation [25]. In this paper we will not worry about this issue and simply assume that the dynamics of the background is completely fixed.
}

luminal dispersion. Moreover, since it seems reasonable to expect that quantum effects will remove the general relativistic singularity, a critical frequency might appear above which no horizon would be experienced at all.

In the analysis that follows we will try to avoid making any further assumptions about the physics near the singularity. For instance we will analyze the characteristics of the radiation at retarded times at which the singularity has not yet formed. Our approach will be based on a derivation of Hawking radiation through the relation between the asymptotically past ray trajectories and the asymptotically future ones in the case of a collapsing configuration. The only assumption about these asymptotic extremities are the standard ones, namely, a Minkowski geometry in the asymptotic past, and flatness at spatial infinity also in the asymptotic future. The language we will use is related to the fluid analogy for black holes, which provides the intuitive picture of the spacetime vacuum flowing into the black hole and getting shredded in its center.

Our main results can be summarized as follows. Three crucial elements distinguish the late-time radiation with superluminal dispersion relations from standard relativistic ones. First, at any instant there will be a critical frequency above which no horizon has yet been experienced. This critical frequency will induce a finite limit in the modes contributing to the radiation, which will therefore have a lower intensity than in the standard case, even if the critical frequency is well above the Planck scale. Second, due to the effective frequency dependence of the horizon, the surface gravity will also become frequency dependent and the radiation will depend on the physics inside the black hole. Unless special conditions are imposed on the profile to ensure that the surface gravity is nonetheless the same for all frequencies below the critical one, the radiation spectrum also undergoes a strong qualitative modification. Depending on the relation between the critical frequency and the Planck scale, the radiation from high frequencies is no longer negligible compared to the lowfrequency thermal part, but can even become dominant. This effect becomes more important with increasing critical frequency. Finally, a third effect is that the radiation will extinguish as time advances.

The remainder of this article has the following structure. In Sec. II, we will describe and motivate the classical geometry of our model and the concrete kinds of profiles that we are interested in. In Sec. III, we will briefly review how Hawking's standard result can be obtained in the formalism of our choice for the case of standard relativistic dispersion relations. This calculation will be adapted in Sec. IV to superluminal dispersion relations, and we will analytically obtain a formula for the late-time radiation for this case. Then, in Sec. V, we will present graphics obtained from this analytic formula by numerical integration. These graphics will illustrate the results mentioned in the previous paragraph, which we will discuss in more depth 
and compare with other results in the recent literature in Sec. VI.

\section{THE CLASSICAL GEOMETRY}

We will study radiation effects in simple $1+$ 1-dimensional collapsing configurations. It is well known that in the Hawking process, most of the radiation comes out in the $s$-wave sector. Hence, a spherically symmetric treatment, effectively $1+1$ dimensional, suffices to capture the most relevant aspects of the process. We will work in Painlevé-Gullstrand coordinates, where

$$
d s^{2}=-\left[c^{2}-v^{2}(t, x)\right] d t^{2}-2 v(t, x) d t d x+d x^{2},
$$

and make the further simplification of taking a constant $c$ (we will always write $c$ explicitly to differentiate it from the frequency/wave number dependent $c_{k}$ that will show up in the presence of a superluminal dispersion relation). In this manner all the information about the configuration is encoded in the single function $v(t, x)$. The PainlevéGullstrand coordinates have the advantage, compared to the Schwarzschild form, of being regular at the horizon. Moreover, they suggest a natural interpretation in terms of the language of acoustic models. In such a model, $c$ represents the speed of sound and $v$ is the velocity of the fluid (which corresponds to the velocity of an observer free-falling into the black hole). Throughout the paper we will consider the fluid to be left-moving, $v \leq 0$, so that the outgoing particles of light/sound move towards the right.

The most relevant aspects of the analysis presented hereafter depend only on the qualitative features of the fluid profile. However, to justify some specific calculations later on it is helpful to use the following concrete profiles, see Figs. 1 and 2 . Consider a velocity profile $\bar{v}(x)$ such that $\bar{v}(x \rightarrow+\infty)=0, \bar{v}(x=0)=-c$ and further decreasing monotonically as $x \rightarrow-\infty$. In the fluid image, the fluid

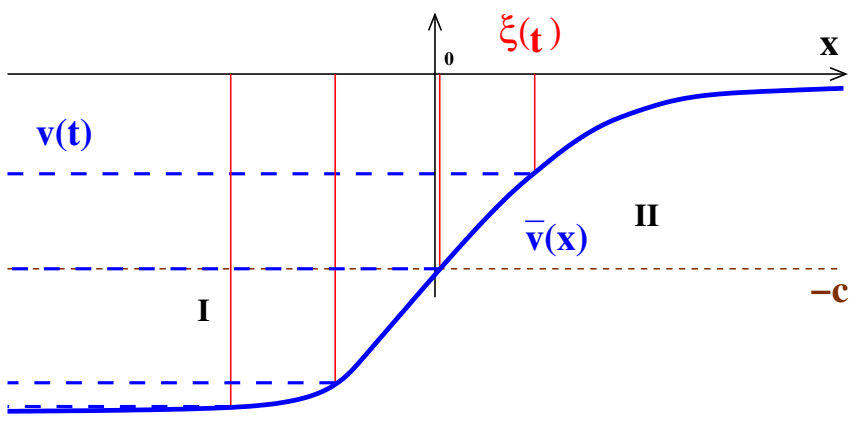

FIG. 1 (color online). Velocity profile of a black hole with a linear slope of the velocity $\bar{v}(x)$, and hence a constant surface gravity $\kappa_{\omega^{\prime}}$, from the classical horizon at $x=0$ down to some predefined limiting value. The auxiliary function $\xi(t)$ separates each instantaneous velocity profile $v(t, x)$ into a dynamical region I: $v(t, x)=v(t)=\bar{v}(\xi(t))$ and a stationary region II: $v(t, x)=\bar{v}(x)$.

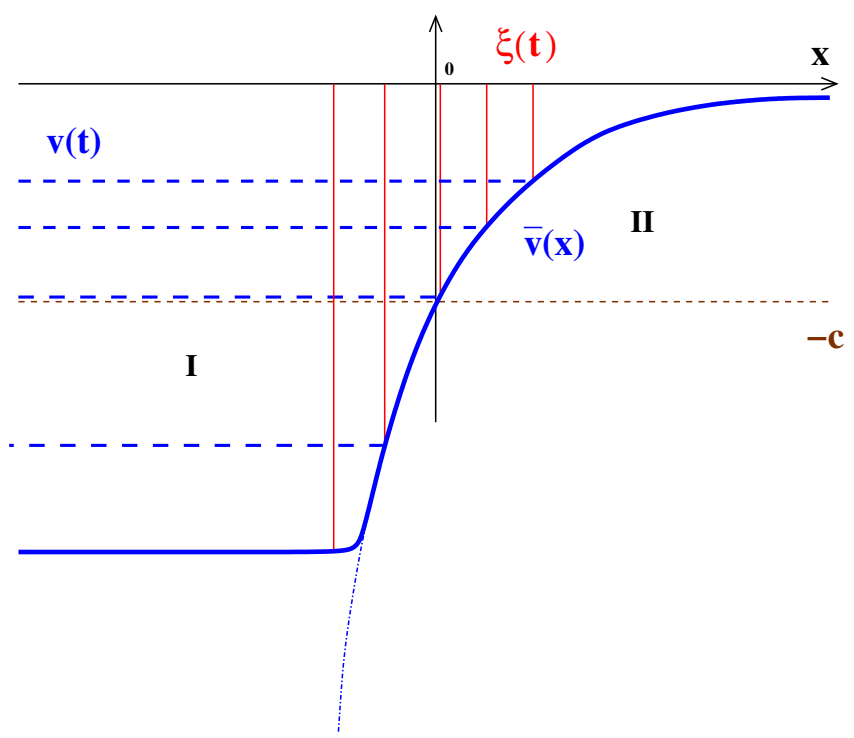

FIG. 2 (color online). Velocity profile characteristic of a Schwarzschild black hole. The slope of $\bar{v}(x)$ increases from the classical horizon at $x=0$ leftwards up to a constant limiting value, leading to a surface gravity $\kappa_{\omega^{\prime}}$ which increases with the frequency.

nearly stands still at large distances and accelerates inwards, with a sonic point or horizon at $x=0$. The further decrease in $\bar{v}$ can either be linear until a constant limiting value is almost achieved, as in Fig. 1, or of the form

$$
\bar{v}(x)=-c \sqrt{\frac{2 M / c^{2}}{x+2 M / c^{2}}}
$$

again up to a constant limiting value, as in Fig. 2. Let us recall that this second velocity profile corresponds to the Schwarzschild line element with $M$ the central mass, as can be seen [35] by reparametrizing the time coordinate and using $r=x+2 M / c^{2}$ as radial coordinate.

Up to here, we have described a stationary profile. Now, to incorporate the dynamics of the collapse, let us introduce a monotonically decreasing function $\xi(t)$, $\xi(t \rightarrow-\infty) \rightarrow+\infty$, and define $v(t, x)$ as

$$
v(t, x)= \begin{cases}\bar{v}(\xi(t)) & \text { if } x \leq \xi(t) \\ \bar{v}(x) & \text { if } x \geq \xi(t)\end{cases}
$$

Imagining the collapse of a homogeneous star, the function $\xi(t)$ represents the distance from the star surface to its gravitational (Schwarzschild) radius. The dynamical configuration that we obtain consists of a series of snapshots. In each snapshot, $|v(t, x)|$ increases (i.e., the fluid accelerates) from $\bar{v}=0$ for $x \rightarrow+\infty$, up to a point $x_{0}=\xi(t)$, and then remains constant as $x$ further decreases towards $-\infty$. For consecutive snapshots, the point $x_{0}=\xi(t)$ moves leftwards, so $v(t, x)$ covers an ever larger part of $\bar{v}(x)$.

From the point of view of an outgoing (right-moving) particle of light/sound, the configuration is nicely split up 


\section{BARCELÓ, L. J. GARAY, AND G. JANNES}

into, first, a dynamical or $t$-dependent region, and then a stationary $x$-dependent region, as defined in (2.3). ${ }^{2}$

The difference between the two types of profiles, Figs. 1 and 2, can best be explained in terms of the surface gravity. When modifying the dispersion relation, the horizon becomes a frequency-dependent concept: each frequency experiences a different horizon, as we will see explicitly in Sec. IV. In particular, for superluminal modifications, the horizon forms later (i.e., at higher values of $|\bar{v}|$, or more negative values of $x$ ) for increasing frequencies. The surface gravity will then also become frequency dependent:

$$
\kappa_{\omega^{\prime}} \equiv c\left|\frac{d \bar{v}}{d x}\right|_{x=x_{H, \omega^{\prime}}},
$$

where $x_{H, \omega^{\prime}}$ is the frequency-dependent location of the horizon. This surface gravity will be seen in Secs. IV and $\mathrm{V}$ to play a crucial role. This explains our choice of the two types of profiles: In our first profile, Fig. 1, we have considered a linear velocity profile $|\bar{v}(x)|$ from the classical or zero-frequency horizon $(c=|v|)$ down to some given limiting value, $|v|_{\max }$, from where it stays constant. This maximum velocity defines a critical frequency $\omega_{c}^{\prime}$ as we will discuss in more detail in Sec. IV. Then the surface gravity $\kappa_{\omega^{\prime}}$ will be frequency-independent up to this critical frequency $\omega_{c}^{\prime}$ after which it will rapidly vanish. In the second profile, see Fig. 2, we have taken a velocity profile typical for a Schwarzschild black hole, and therefore $\kappa_{\omega^{\prime}}$ increases with $\omega^{\prime}$, again up to a predefined limiting value corresponding to the horizon for a critical frequency $\omega_{c}^{\prime}$. In the context of the fluid analogy, it seems obvious that some mechanism will avoid the formation of a singularity. But in any case, we will also take into account the limit for an infinite critical frequency, which corresponds to a velocity profile with $|v|_{\max } \rightarrow \infty$.

\section{STANDARD DISPERSION RELATIONS}

In this section we will briefly review a way of deriving Hawking's formula for the radiation of a black hole with standard (relativistic) dispersion relations of the form $\omega^{2}=c^{2} k^{2}$. For the sake of simplicity, we will only consider a massless scalar field. We will summarize the main steps: from the Klein-Gordon inner product and the definition of the Bogoliubov $\beta$ coefficients, over the relation between past and future null coordinates, to the blackbody radiation in the wave packet formulation. Our aim is to present the key points of the procedure in such a way that they can easily be adapted to superluminally modified dispersion relations - the subject of the next sections.

\footnotetext{
${ }^{2}$ In a physically realistic model, the apparent kink in the profile where the transition between both regions takes place will of course be smoothed out.
}

PHYSICAL REVIEW D 79, 024016 (2009)

\section{A. Inner product}

The d'Alembertian or wave equation for a massless scalar field in $1+1$ dimensions for the metric (2.1) with constant $c$ can be written as

$$
\left(\partial_{t}+\partial_{x} v\right)\left(\partial_{t}+v \partial_{x}\right) \phi=c^{2} \partial_{x}^{2} \phi .
$$

This conformally invariant theory is equivalent to the dimensionally reduced $3+1$ spherically symmetric theory if one neglects the backscattering due to the angularmomentum potential barrier (responsible for the so-called grey-body factors).

In the space of solutions of this wave equation, we can define the Klein-Gordon pseudoscalar product

$$
\left(\varphi_{1}, \varphi_{2}\right) \equiv-i \int_{\Sigma} d \Sigma^{\mu} \varphi_{1} \overleftrightarrow{\mathrm{\partial}}_{\mu} \varphi_{2}^{*}
$$

which is independent of the choice of the spatial slice $\Sigma$. For a $t=$ constant slice, and, in particular, for $t \rightarrow+\infty$, this becomes

$\left(\varphi_{1}, \varphi_{2}\right)=-i \int d x\left[\varphi_{1}\left(\partial_{t}+v \partial_{x}\right) \varphi_{2}^{*}-\varphi_{2}^{*}\left(\partial_{t}+v \partial_{x}\right) \varphi_{1}\right]$

We can define future null coordinates $u(t, x)$ and $w(t, x)$, such that, when $t, x \rightarrow+\infty$,

$$
u(t, x) \rightarrow t-x / c, \quad w(t, x) \rightarrow t+x / c .
$$

Writing the inner product in terms of these null coordinates gives, for the limit $t \rightarrow+\infty$,

$$
\begin{aligned}
\left(\varphi_{1}, \varphi_{2}\right)= & -\frac{i c}{2}\left\{\int_{-\infty}^{+\infty} d u\left[\varphi_{1} \partial_{u} \varphi_{2}^{*}-\varphi_{2}^{*} \partial_{u} \varphi_{1}\right]_{w=+\infty}\right. \\
& \left.+\int_{-\infty}^{+\infty} d w\left[\varphi_{1} \partial_{w} \varphi_{2}^{*}-\varphi_{2}^{*} \partial_{w} \varphi_{1}\right]_{u=+\infty}\right\} .
\end{aligned}
$$

Note that, in the derivation of this formula, we have only made use of coordinate transformations, without any appeal to their null character or to geometrical tools such as conformal diagrams or the deformation of Cauchy surfaces. This is important because in the case of modified dispersion relations, such geometrical concepts become problematic and actually can only be maintained in the context of a rainbow geometry-if at all.

Similarly we could have used past null coordinates $(U, W)$, which obey, when $t \rightarrow-\infty$,

$$
U(t, x) \rightarrow t-x / c, \quad W(t, x) \rightarrow t+x / c,
$$

to calculate the inner product in the asymptotic past.

\section{B. Bogoliubov $\boldsymbol{\beta}$ coefficients}

The right-moving positive-energy solutions associated with the asymptotic past and with the asymptotic future, normalized in the Dirac-delta sense, can be expressed as 


$$
\psi_{\omega^{\prime}}^{\prime}=\frac{1}{\sqrt{2 \pi c \omega^{\prime}}} e^{-i \omega^{\prime} U}, \quad \psi_{\omega}=\frac{1}{\sqrt{2 \pi c \omega}} e^{-i \omega u},
$$

respectively, where we use primes to indicate asymptotic past values.

The relevant Bogoliubov $\beta$ coefficients encoding the production of radiation are defined as $\beta_{\omega \omega^{\prime}} \equiv\left(\psi_{\omega^{\prime}}^{\prime}, \psi_{\omega}^{*}\right)$. The mode mixing relevant for the Hawking process occurs in the right-moving sector. Therefore, we only need to calculate the first term in the scalar product (3.5). Then, plugging (3.7) into the definition of $\beta$ and integrating by parts we obtain the simple expression

$$
\beta_{\omega \omega^{\prime}}=\frac{1}{2 \pi} \sqrt{\frac{\omega}{\omega^{\prime}}} \int d u e^{-i \omega^{\prime} U(u)} e^{-i \omega u},
$$

so that all the information about the produced radiation is contained in the relation $U=U(u) \equiv U(u, w \rightarrow+\infty)$.

\section{Relation $U(u)$}

For a standard relativistic dispersion relation, it is well known that the relation between $U$ and $u$ for a configuration that forms a horizon (in our case at $x=0$ ) can be expressed at late times as $U=U_{H}-A e^{-\kappa u / c}$ (note that we use a subscript $H$ for all quantities associated with the horizon), where $U_{H}, A$ and the surface gravity

$$
\kappa \equiv c\left|\frac{d \bar{v}}{d x}\right|_{x=0}
$$

are constants.

We can define a threshold time $u_{I}$ at which an asymptotic observer will start to detect thermal radiation from the black hole. This retarded time corresponds to the moment at which the function $U(u)$ enters the exponential regime. We can then rewrite the previous expression, valid for $u>u_{I}$, as

$$
U=U_{H}-A_{0} e^{-\kappa\left(u-u_{I}\right) / c} .
$$

Plugging this relation into (3.8) and integrating in $u$ gives

$$
\begin{aligned}
\beta_{\omega \omega^{\prime}}= & \frac{1}{2 \pi} \sqrt{\frac{\omega}{\omega^{\prime}}} \frac{c}{\kappa} \exp \left[-i \omega^{\prime} U_{H}\right] \exp \left[-i \frac{c \omega}{\kappa} \ln \left(\omega^{\prime} A_{0}\right)\right] \\
& \times \exp \left(-i \omega u_{I}\right) \exp \left(-\frac{\pi c \omega}{2 \kappa}\right) \Gamma(i c \omega / \kappa) .
\end{aligned}
$$

\section{Wave packet formulation}

In order to obtain physically sensible results, it is a good precautionary measure to replace the monochromatic rays described until now by wave packets (see e.g. [11] or the discussion in [36]). Positive-energy wave packets can be defined as

$$
P_{\omega_{j}, u_{l}}(\omega) \equiv \begin{cases}\frac{e^{i \omega u_{l}}}{\sqrt{\Delta \omega}} & -\frac{1}{2} \Delta \omega<\omega-\omega_{j}<\frac{1}{2} \Delta \omega \\ 0 & \text { otherwise }\end{cases}
$$

where $u_{l} \equiv u_{0}+2 \pi l / \Delta \omega$ with $u_{0}$ an overall reference and $l$ an integer phase parameter. The central frequencies of the wave packets are $\omega_{j} \equiv j \Delta \omega$, with $\Delta \omega$ their width.

Then, from the expression $\beta_{\omega_{j}, u_{l} ; \omega^{\prime}} \equiv \int d \omega \beta_{\omega \omega^{\prime}} P_{\omega_{j}, u_{l}}(\omega)$, and assuming that the wave packets are sufficiently narrow $\left(\Delta \omega \ll \omega_{j}\right)$, we obtain

$$
\left|\beta_{\omega_{j}, u_{l} ; \omega^{\prime}}\right|^{2} \approx \frac{c \Delta \omega}{2 \pi \omega^{\prime} \kappa} \frac{\sin ^{2}\left(z-z_{l}\right)}{\left(z-z_{l}\right)^{2}} \frac{1}{\exp \left(\frac{2 \pi c \omega_{j}}{\kappa}\right)-1},
$$

where we have defined

$$
z=\frac{c \Delta \omega}{2 \kappa} \ln \omega^{\prime} A_{0}, \quad z_{l}=\frac{\Delta \omega}{2}\left(u_{l}-u_{I}\right) .
$$

Finally, integration in $\omega^{\prime}$ gives the number of particles with frequency $\omega_{j}$ detected at time $u_{l}$ by an asymptotic observer:

$$
\begin{gathered}
N_{\omega_{j}, u_{l}}=\int_{0}^{+\infty} d \omega^{\prime}\left|\beta_{\omega_{j}, u_{l} ; \omega^{\prime}}\right|^{2} \\
\approx \frac{1}{\exp \left(2 \pi c \omega_{j} / \kappa\right)-1},
\end{gathered}
$$

which reproduces Hawking's formula (in the absence of backscattering) and corresponds to a Planckian spectrum with temperature $T_{H}=\kappa /(2 \pi c)$.

\section{SUPERLUMINALLY MODIFIED DISPERSION RELATIONS}

In this section we will indicate how the late-time radiation originating from the formation of a black hole from collapse can be calculated in the case of superluminal dispersion relations.

We introduce superluminally modified dispersion relations by adding a quartic term to the wave equation:

$$
\left(\partial_{t}+\partial_{x} v\right)\left(\partial_{t}+v \partial_{x}\right) \phi=c^{2}\left(\partial_{x}^{2}+\frac{1}{k_{P}^{2}} \partial_{x}^{4}\right) \phi,
$$

where $k_{P}$ (the "Planck scale") is the scale at which nonrelativistic deviations in the associated dispersion relation

$$
(\omega-v k)^{2}=c^{2} k^{2}\left(1+\frac{k^{2}}{k_{P}^{2}}\right)
$$

become significant. We use this relation for concreteness and because it is the one that shows up in Bose-Einstein condensates, where $k_{P}=2 / \xi$, with $\xi$ the healing length of the condensate. However, qualitatively, our results will not depend on the specific form of the deviations from the relativistic dispersion relation but on their superluminal character.

This dispersion relation leads to a modification in both the phase velocity $v_{\mathrm{ph}}$ and the group velocity $v_{g}$. For a right-moving wave, we have 


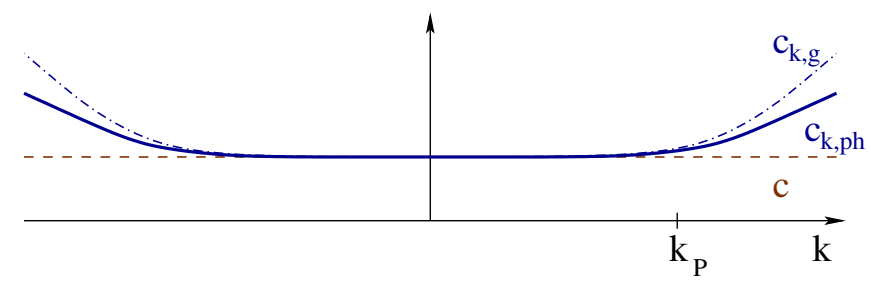

FIG. 3 (color online). Behavior of the effective phase $c_{k \text {,ph }}$ and group $c_{k, g}$ speeds of light/sound with respect to the wave number $k$. Because of the nonrelativistic dispersion relation, the effective velocities become "superluminal" for $k>k_{P}$ (where $k_{P}$ is the Planck scale).

$$
v_{\mathrm{ph}} \equiv \frac{\omega}{k}=c_{k, \mathrm{ph}}+v, \quad v_{g} \equiv \frac{d \omega}{d k}=c_{k, g}+v,
$$

where we have introduced the effective $k$-dependent phase and group speeds of light/sound

$$
c_{k, \mathrm{ph}}=c \sqrt{1+\frac{k^{2}}{k_{P}^{2}}}, \quad c_{k, g}=c \frac{1+2 \frac{k^{2}}{k_{P}^{2}}}{\sqrt{1+\frac{k^{2}}{k_{P}^{2}}}}
$$

respectively (see Fig. 3). Both $c_{k \text {,ph }}$ and $c_{k, g}$ become larger than $c$ (i.e., become "superluminal") as $k$ increases above $k_{P}$.

At first sight, it seems obvious that the ray equation should be defined in terms of the group velocity $v_{g}$. Nevertheless, the question of whether the velocity relevant for Hawking radiation is the group or the phase velocity seems to be tricky $[22,37,38]$. For example, (4.9) below suggests that the phase velocity might be relevant. We limit ourselves to remark that $c_{k, g}$ and $c_{k, \mathrm{ph}}$ show the same qualitative behavior. Hence our results are independent of this question and we will simply write $c_{k}$ [or $c_{k\left(\omega^{\prime}\right)}$ when wishing to emphasize the frequency dependence]. Then, there will be a horizon, which becomes frequency dependent, when $c_{k}+v=0$, irrespectively of whether $c_{k, g}$ or $c_{k \text {,ph }}$ is used for $c_{k}$. Moreover, since $c_{k}$ becomes arbitrarily high for increasing wave number $k$, given a certain $|v|_{\max }$ at a particular instant of time, there will be a critical $\omega_{c}^{\prime}$ such that waves with an initial frequency $\omega^{\prime}>\omega_{c}^{\prime}$ do not experience a horizon at all. The only exception to this rule occurs when the velocity profile ends in a singularity $\bar{v} \rightarrow-\infty$, which implies $\omega_{c}^{\prime} \rightarrow \infty$.

Our aim is to calculate the black hole radiation with superluminally modified dispersion relations. We will now repeat the main steps of Sec. III and point out where and how these modifications must be taken into account.

\section{A. Generalization of inner product}

The essential point with regard to the pseudoscalar product (3.3) is the following. Its explicit form for $t=$ constant is not changed by the presence of the $\partial_{x}^{4}$ term in the wave equation (see also the discussion in [25]). Indeed, it is still a well-defined inner product, and, in particular, it is still a conserved quantity, since

$$
\partial_{t}\left(\varphi_{1}, \varphi_{2}\right)=\int d x \varphi_{1} \overleftrightarrow{\partial}_{x}^{4} \varphi_{2}^{*}
$$

which can be seen, by repeated integration by parts, to vanish under the usual assumption that the fields die off asymptotically. Note that the modification of the dispersion relation singles out a preferred time frame: the "laboratory" time $t$. Changing to another time $\tilde{t}$ will in general lead to a mixing between $t$ and $x$, and hence the simple relations given here would no longer be valid.

Using the preferred time $t$, and making exactly the same change of coordinates as in the case of standard dispersion relations, we can again transform the inner product, evaluated at $t \rightarrow+\infty$, into the expression (3.5) in terms of $u$ and $w$. As in the standard case, only the first term is relevant for the Hawking process:

$$
-\frac{i c}{2} \int_{-\infty}^{+\infty} d u\left[\varphi_{1} \partial_{u} \varphi_{2}^{*}-\varphi_{2}^{*} \partial_{u} \varphi_{1}\right]_{w=+\infty}
$$

Note that we are now using $u$ and $w$ merely as a perfectly good set of (auxiliary) coordinates, in order to cast the inner product into a useful form. However, we cannot associate with them any null character such as they had in the case of standard dispersion relations.

\section{B. Rainbow null coordinates}

Let us define some sets of spacetime functions that will prove to be useful in what follows. Given a fluid profile of the type described in Sec. II, we can integrate the ray equation

$$
\frac{d x}{d t}=c_{k\left(\omega^{\prime}\right)}(t, x)+v(t, x),
$$

starting from the past left infinity towards the right. The ray initially has a frequency $\omega^{\prime}$ and an associated initial wave number $k^{\prime}$, from which we can deduce the value of $c_{k^{\prime}}$. In the left region, where the velocity profile is dynamic but position-independent $[v(x, t)=v(\xi(t))$, with $\xi(t)$ the auxiliary function introduced in Sec. II], $k=k^{\prime}$ can be considered as fixed while the frequency changes [this is what happens in a mode solution of Eq. (4.1) in this region]. Then, we can define the function

$$
\mathcal{U}_{\omega^{\prime}}(t, x)=\frac{1}{\omega^{\prime}} \int \bar{\omega}(t) d t-\frac{k^{\prime}}{\omega^{\prime}} x,
$$

where $\bar{\omega}(t)$ is the instantaneous frequency of the particle at each time $t$, defined through the dispersion relation and such that $\bar{\omega}(t \rightarrow-\infty)=\omega^{\prime}$.

When the ray reaches the kink it passes into a stationary region in which the velocity profile only depends on the position $[v(t, x)=\bar{v}(x)]$. At the kink, the ray still has the initial wave number $k^{\prime}$ and a frequency $\omega$. In its propagation towards the right this frequency now remains fixed 
while its wave number becomes a decreasing function $\bar{k}(x)$ of the position, such that $\bar{k}\left(\xi\left(t_{0}\right)\right)=k^{\prime}$, with $t_{0}$ the moment at which the kink is crossed. The final frequency of the ray will then simply be $\omega$ and its final wave number $k=\lim _{x \rightarrow+\infty} \bar{k}(x)$. In this region, then, the function $\mathcal{U}_{\omega^{\prime}}(t, x)$ can be expressed as

$$
\mathcal{U}_{\omega^{\prime}}(t, x)=t-\int \frac{\bar{k}(x)}{\omega} d x .
$$

Note here that $\omega$ and $\bar{k}(x)$ both depend on the initial $\omega^{\prime}$.

The same can be done by integrating the ray equation starting from the future. In this way we can define $u_{\omega}$ functions. (The same procedure can be used to define $\mathcal{W}_{\omega^{\prime}}$ and $\left.w_{\omega}\right)$. It is worth noting that $\mathcal{U}_{\omega^{\prime}}$ and $u_{\omega}$ are not null coordinates in the usual geometric sense, since they are frequency dependent, but they nevertheless share many properties with null coordinates.

\section{Bogoliubov $\boldsymbol{\beta}$ coefficient}

The calculation of the inner product (4.6) involves the limit $w \rightarrow+\infty$ (equivalently, $w_{\omega} \rightarrow+\infty$ ). So, the general form

$$
u_{\omega}=u_{\omega}(u, w)
$$

can be simplified to $u_{\omega}=u_{\omega}(u) \equiv u_{\omega}(u, w \rightarrow+\infty)$. We can therefore change variables in the inner product from $u$ to $u_{\omega}$. The combination of the derivative and the integral means that the form of (4.6) is preserved in the new integration variable $u_{\omega}$. Then we can write the Bogoliubov $\beta$ coefficients relevant for the Hawking process as

$$
\beta_{\omega \omega^{\prime}}=-\frac{i c}{2} \int_{-\infty}^{+\infty} d u_{\omega}\left[\psi_{\omega^{\prime}}^{\prime} \partial_{u_{\omega}} \psi_{\omega}-\psi_{\omega} \partial_{u_{\omega}} \psi_{\omega^{\prime}}^{\prime}\right]_{w_{\omega}=+\infty}
$$

Now, assuming that the profiles vary slowly (in scales much larger than the Planck distance), the right-moving positive-energy modes associated with past and future infinity can be approximated by the following simple expressions:

$$
\begin{aligned}
\psi_{\omega^{\prime}}^{\prime} & \approx \frac{1}{\sqrt{2 \pi c \omega^{\prime}}} e^{-i \omega^{\prime} u_{\omega^{\prime}},} \\
\psi_{\omega} & \approx \frac{1}{\sqrt{2 \pi c \omega}} e^{-i \omega u_{\omega}},
\end{aligned}
$$

so that the Bogoliubov coefficients read

$$
\beta_{\omega \omega^{\prime}} \approx \frac{1}{2 \pi} \sqrt{\frac{\omega}{\omega^{\prime}}} \int d u_{\omega} e^{-i \omega^{\prime} u_{\omega^{\prime}}\left(u_{\omega}\right)} e^{-i \omega u_{\omega}} .
$$

In analogy with the standard case, all information about the radiation is seen to be encoded in the relation $\mathcal{U}_{\omega^{\prime}}\left(u_{\omega}\right)$. In this expression $\omega^{\prime}$ is the initial frequency of a ray at the past left infinity and $\omega=\omega\left(\omega^{\prime}\right)$ its final frequency when reaching the future right infinity.

The previous approximation for the Bogoliubov coefficients amounts to considering profiles that vary slowly both with $x$ and $t$. This is equivalent to considering large black holes. In general the quartic term in the wave equation, or equivalently the quartic modification of the dispersion relation, introduce a new source of backscattering, on top of the usual angular-momentum potential barrier which we have already neglected. In our approximation, this additional backscattering (beyond the standard grey-body factors) has been neglected. For large black holes this contribution will in any case be very small as has been observed in numerical simulations [22]. In addition we are also neglecting any reflection caused by the kink. However, let us remark that given an approximative scheme for calculating $\mathcal{U}_{\omega^{\prime}}\left(u_{\omega}\right)$ for general profiles $v(t, x)$, the same $\mathcal{U}_{\omega^{\prime}}\left(u_{\omega}\right)$ obtained from a profile with a kink would be obtainable from one (or several) specific $v(t, x)$, this time perfectly smooth and thus causing no further backscattering. Our results, which rely only on the specific form of the relation $\mathcal{U}_{\omega^{\prime}}\left(u_{\omega}\right)$, are therefore valid beyond the specific configurations with a kink presented in this paper.

\section{Relation $\mathcal{U}_{\boldsymbol{\omega}^{\prime}}\left(\boldsymbol{u}_{\boldsymbol{\omega}}\right)$}

Our next task is to calculate the uniparametric family of functions $\mathcal{U}_{\omega^{\prime}}$ and the relation between $\mathcal{U}_{\omega^{\prime}}$ and $u_{\omega}$ for different configurations. As explained in Sec. IV B, the relation $\mathcal{U}_{\omega^{\prime}}\left(u_{\omega}\right)$ is obtained by integrating the ray equation $d x / d t=c_{k}+v$ using the profiles discussed in Sec. II. These can be described by means of stationary profiles $\bar{v}(x)$ and an auxiliary function $\xi(t)$; see the definition of $v(t, x)$ in (2.3). We will use a straightforward extension of the procedure established in [39] (see also [40] for a summary) for a relativistic dispersion relation. Care must be taken, however, with the quantities that depend on the frequency $\omega^{\prime}$. In particular,

(i) we will denote by $x_{H, \omega^{\prime}}$ and $t_{H, \omega^{\prime}}$ the position and the time at which the horizon associated with a particular initial frequency $\omega^{\prime}$ is formed;

(ii) the surface gravity $\kappa_{\omega^{\prime}}$, defined in (2.4), allows one to write, for all $\omega^{\prime}<\omega_{c}^{\prime}$ and for $x$ close to $x_{H, \omega^{\prime}}$ :

$$
\bar{v}(x) \approx-c_{k}+\frac{1}{c} \kappa_{\omega^{\prime}}\left(x-x_{H, \omega^{\prime}}\right),
$$

up to higher-order terms in $x-x_{H, \omega^{\prime}}$;

(iii) the linearization of $\xi(t)$ near $t_{H, \omega^{\prime}}$ also requires the introduction of an $\omega^{\prime}$-dependent parameter $\lambda_{\omega^{\prime}}$ :

$$
\xi(t) \approx x_{H, \omega^{\prime}}-\lambda_{\omega^{\prime}}\left(t-t_{H, \omega^{\prime}}\right),
$$

again up to higher-order terms.

Let us consider laboratory times $t>t_{H, 0} \equiv t_{H, \omega^{\prime}=0}$ such that $\xi(t)$ has already crossed the classical horizon at $x=0$ : $\xi\left(t>t_{H, 0}\right)<0$. As we explained earlier we can define a 


\section{BARCELÓ, L. J. GARAY, AND G. JANNES}

critical frequency $\omega_{c}^{\prime}$ as the minimum initial frequency such that $c_{k}+\bar{v}(\xi(t))>0$ for all $\omega^{\prime}>\omega_{c}^{\prime}$, or, in other words, the minimum frequency which at that particular time has not experienced any horizon yet.

In the dynamical part of the profile, where $v=\bar{v}(\xi(t))$, for rays crossing the kink just before the formation of the horizon, integration of the ray equation leads to

$$
\mathcal{U}_{\omega^{\prime}} \approx \mathcal{U}_{H, \omega^{\prime}}+\frac{\lambda_{\omega^{\prime}}}{c_{k^{\prime}}}\left(t_{0, \omega^{\prime}}-t_{H, \omega^{\prime}}\right),
$$

for small values of $\left|t_{0, \omega^{\prime}}-t_{H, \omega^{\prime}}\right|$ (see [39] for details of the calculation in the case of a relativistic dispersion relation), where $t_{0, \omega^{\prime}}$ is the (frequency-dependent) time at which the kink separating the dynamical and the stationary regions is crossed, $c_{k^{\prime}}=\lim _{t \rightarrow-\infty} c_{k\left(\omega^{\prime}\right)}$ is the speed of light in the asymptotic past, and

$$
\mathcal{U}_{H, \omega^{\prime}} \equiv t_{H, \omega^{\prime}}-\frac{\xi\left(t_{H, \omega^{\prime}}\right)}{c_{k^{\prime}}}+\frac{1}{c_{k^{\prime}}} \int_{-\infty}^{t_{H, \omega^{\prime}}} \bar{v}(\xi(t)) d t
$$

is the ray constituting the horizon associated with the frequency $\omega^{\prime}$.

In the stationary part of the profile, where $v=\bar{v}(x)$, we obtain, for $t_{0, \omega^{\prime}} \rightarrow t_{H, \omega^{\prime}}$, again using a method and notation based on [39],

$$
u_{\omega} \approx t_{0, \omega^{\prime}}-\frac{\xi\left(t_{0, \omega^{\prime}}\right)}{c_{k, f}}+C_{1}-\frac{c}{\kappa_{\omega^{\prime}}} \ln \left[\xi\left(t_{0, \omega^{\prime}}\right)-x_{H, \omega^{\prime}}\right],
$$

where $c_{k, f}=\lim _{t \rightarrow+\infty} c_{k\left(\omega^{\prime}\right)}$ is the speed of light in the asymptotic future, and $C_{1}$ a bulk integration constant. So

$$
\xi\left(t_{0, \omega^{\prime}}\right)-x_{H, \omega^{\prime}}=C_{\omega^{\prime}} e^{-\kappa_{\omega^{\prime}} u_{\omega} / c} .
$$

Both regions are connected by combining (4.17) and (4.20). Making use of (4.16), this gives

$$
\mathcal{U}_{\omega^{\prime}}=\mathcal{U}_{H, \omega^{\prime}}-A_{\omega^{\prime}} e^{-\kappa_{\omega^{\prime}} u_{\omega} / c} .
$$

This relation is valid for frequencies for which a horizon is experienced, i.e. for $\omega^{\prime}<\omega_{c}^{\prime}$ and times $u_{\omega}>u_{I, \omega^{\prime}}$, where $u_{I, \omega^{\prime}}$ is the threshold time defined in Sec. IIIC (which, unsurprisingly, has become frequency dependent). Again, as in the case of standard dispersion relationsSec. III C-we can write

$$
\mathcal{U}_{\omega^{\prime}}=\mathcal{U}_{H, \omega^{\prime}}-A_{0} e^{-\kappa_{\omega^{\prime}}\left(u_{\omega}-\bar{u}_{I, \omega^{\prime}}\right) / c},
$$

where $\bar{u}_{I, \omega^{\prime}}$ is essentially $u_{I, \omega^{\prime}}$ (with possible higher-order corrections). Assuming that the collapse takes place rapidly, it is a good approximation to replace $\bar{u}_{I, \omega^{\prime}}$ by $\bar{u}_{I, \omega_{c}^{\prime}}$. Actually, as we will see shortly, this is a conservative estimate, in the sense that it slightly underestimates the superluminal correction to the radiation spectrum.
PHYSICAL REVIEW D 79, 024016 (2009)

\section{E. Wave packet formulation}

The relation $\mathcal{U}_{\omega^{\prime}}\left(u_{\omega}\right)$ we are considering interpolates between a linear behavior at early times and an exponential behavior at late times. It has the same form as the relation $U(u)$ for standard dispersion relations, and so we can continue following the steps of the standard case. In particular, the equivalent of (3.11) is obtained by integrating out $u_{\omega}$. Note that we use the subscript $\omega$ to emphasize that the $u_{\omega}$ are not the null coordinates of the standard case, but this should not be interpreted as an explicit function of $\omega$ and so does not complicate the integration steps in $u_{\omega}$ and $\omega$. However, when integrating $\left|\beta_{\omega_{j}, u_{l} ; \omega^{\prime}}\right|^{2}$ in $\omega^{\prime}$ to obtain $N_{\omega_{j}, u_{l}}$, see Sec. IIID, we must carefully consider the frequency dependence of the relevant terms, i.e., of $\mathcal{U}_{H, \omega^{\prime}}, \bar{u}_{I, \omega^{\prime}}$, and $\kappa_{\omega^{\prime}}$. The term carrying $\mathcal{U}_{H, \omega^{\prime}}$ is moduloed away in (3.13), and we have replaced $\bar{u}_{I, \omega^{\prime}}$ by $\bar{u}_{I, \omega_{c}^{\prime}}$, so the only relevant frequency-dependent factor that we are left with is the surface gravity $\kappa_{\omega^{\prime}}$.

Moreover, because of the critical frequency $\omega_{c}^{\prime}$ in the horizon formation process, a finite upper boundary will also be induced in the integral. Indeed, frequencies $\omega^{\prime}>\omega_{c}^{\prime}$ do not contribute to the radiation at all, since they do not experience a horizon. This is a delicate but crucial point. It was already observed long ago by Jacobson [14] that trying to solve the trans-Planckian problem naively by imposing a cutoff frequency would seemingly extinguish Hawking radiation on a relatively short time scale. In our case, however, this cutoff is not imposed ad hoc, but appears explicitly because of the superluminal character of the system at high frequencies. Moreover, the critical frequency, and hence the upper boundary induced in the integral, depend directly on the physics inside the horizon. Indeed, given a certain velocity profile, and, in particular, its behavior near the center of the black hole, the critical frequency can be calculated by setting $c_{k}=|v|$ in Eq. (4.4) and extracting the corresponding critical frequency from the dispersion relation (4.2). We will see this effect graphically in Sec. V.

In analogy with Eqs. (3.13) and (3.15), we now obtain the number of particles detected for each frequency $\omega_{j}$ as

$$
\begin{aligned}
N_{\omega_{j}, u_{l}}= & \int_{0}^{\omega_{c}^{\prime}} d \omega^{\prime}\left|\beta_{\omega_{j}, u_{l} ; \omega^{\prime}}\right|^{2} \\
\approx & \frac{c \Delta \omega}{2 \pi} \int_{0}^{\omega_{c}^{\prime}} \frac{d \omega^{\prime}}{\omega^{\prime}} \frac{1}{\kappa_{\omega^{\prime}}} \frac{\sin ^{2}\left[\frac{\kappa_{0}}{\kappa_{\omega^{\prime}}}\left(z-z_{l, \omega^{\prime}}\right)\right]}{\left[\frac{\kappa_{0}}{\kappa_{\omega^{\prime}}}\left(z-z_{l, \omega^{\prime}}\right)\right]^{2}} \\
& \times \frac{1}{\exp \left(\frac{2 \pi c \omega_{j}}{\kappa_{\omega^{\prime}}}\right)-1},
\end{aligned}
$$

where now $z=\frac{c \Delta \omega}{2 \kappa_{0}} \ln \omega^{\prime} A_{0}$, with $\kappa_{0} \equiv \kappa_{\omega^{\prime}=0}$ [which corresponds to the standard $\kappa$ of (3.9)], and $z_{l, \omega^{\prime}}=\frac{\kappa_{\omega^{\prime}}}{\kappa_{0}} \frac{\Delta \omega}{2} \times$ $\left(u_{l}-\bar{u}_{I, \omega_{c}^{\prime}}\right)$. Changing the integration variable from $\omega^{\prime}$ to $z$, we finally obtain the central expression in our analysis: 


$$
\begin{aligned}
N_{\omega_{j}, u_{l}}= & \frac{1}{\pi} \int_{-\infty}^{z_{c}} d z \frac{\kappa_{0}}{\kappa_{\omega^{\prime}}} \frac{\sin ^{2}\left[\frac{\kappa_{0}}{\kappa_{\omega^{\prime}}}\left(z-z_{l, \omega^{\prime}}\right)\right]}{\left[\frac{\kappa_{0}}{\kappa_{\omega^{\prime}}}\left(z-z_{l, \omega^{\prime}}\right)\right]^{2}} \\
& \times \frac{1}{\exp \left(\frac{2 \pi c \omega_{j}}{\kappa_{\omega^{\prime}}}\right)-1} .
\end{aligned}
$$

Note that, as we indicated earlier, the use of $\bar{u}_{I, \omega_{c}^{\prime}}$ in the definition of $z_{l, \omega^{\prime}}$ is a conservative stance. Indeed, strictly speaking, we should write $z_{l, \omega^{\prime}} \propto\left(u_{l}-\bar{u}_{I, \omega^{\prime}}\right)$. For a fixed $z_{c}$, a smaller value of $z_{l, \omega^{\prime}}$, and hence a larger value of $\bar{u}_{I, \omega^{\prime}}$, means that a larger part of the central peak of the integrand will be integrated over. Since we are replacing $\bar{u}_{I, \omega^{\prime}}$ by the upper bound $\bar{u}_{I, \omega_{c}^{\prime}}$, we are overestimating the resulting radiation (i.e., underestimating the modification with respect to the standard Hawking radiation).

The expression (4.24) brings out the two crucial factors mentioned earlier, and a third, corollary one.

(i) First, it shows the dependence of the total radiation on the critical frequency $\omega_{c}^{\prime}$ (through the integration boundary $z_{c}$ induced by it), as discussed just before obtaining formula (4.23).

(ii) Second, it shows the importance of the frequencydependent $\kappa_{\omega^{\prime}}$ (to be compared with the fixed $\kappa$ of the standard case). Given a concrete profile $v(t, x)$, the frequency dependence of $\kappa_{\omega^{\prime}}$ can be derived explicitly, as we will discuss next.

(iii) Finally, as a corollary of the first point, it shows that, as $u_{l}$ (and hence $z_{l, \omega^{\prime}}$ ) increases, a smaller part of the central peak of the integrand will be integrated over, so the radiation will die off as $u_{l}$ advances.

\section{F. Surface gravity}

A careful analysis has shown that, so far, most formulas for the standard case could be adapted to superluminal dispersion relations by replacing the relevant magnitudes with their frequency-dependent counterparts. For example, $\beta_{\omega \omega^{\prime}}$ was obtained by replacing $U$ and $u$ by $\mathcal{U}_{\omega^{\prime}}$ and $u_{\omega}$, and, in particular $U_{H}, u_{I}$, and $\kappa$ by $\mathcal{U}_{H, \omega^{\prime}}, \bar{u}_{I, \omega^{\prime}}$, and $\kappa_{\omega^{\prime}}$, respectively. Given a concrete profile, we can explicitly deduce the relation between $\kappa_{\omega^{\prime}}$ and $\omega^{\prime}$, with the Planck scale $k_{P}$ as a parameter, as follows. The horizon for a particular initial frequency $\omega^{\prime}$ is formed when

$$
1+\frac{k_{H}^{2}}{k_{P}^{2}}=\frac{\left|v\left(x_{H, \omega^{\prime}}\right)\right|^{2}}{c^{2}},
$$

i.e., when $v_{\mathrm{ph}}=c_{k}+v=0$, where we have used the phase velocity for concreteness. But again, qualitatively, our results would be similar if taking the group velocity $v_{g}$ instead of $v_{\text {ph }}$.

Taking into account that $k_{H}=k^{\prime}$, the dispersion relation in the asymptotic past can be written as

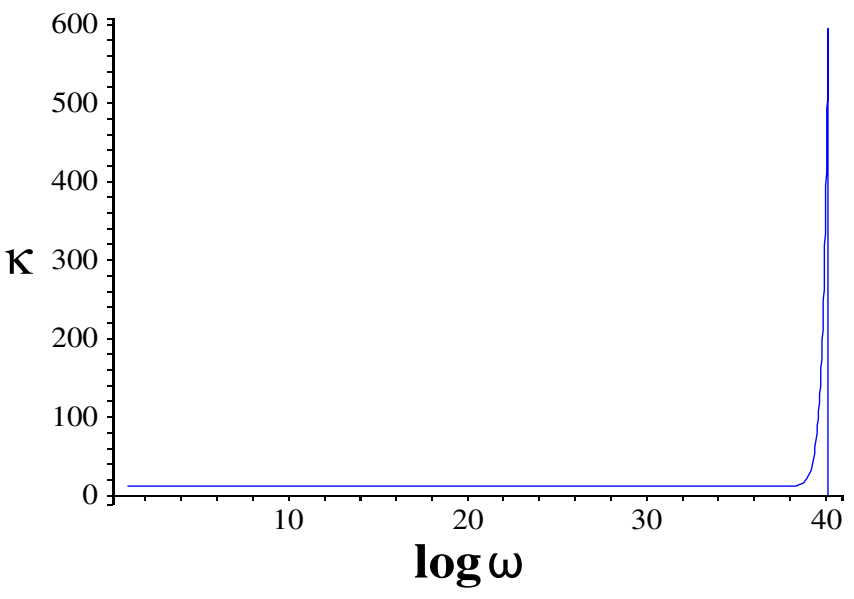

FIG. 4 (color online). Surface gravity $\kappa_{\omega^{\prime}}$ with respect to the (logarithm of the) frequency for a Schwarzschild-type black hole as in Fig. 2, with the Planck scale $k_{P}=10^{39} \mathrm{t}^{-1}$ and the critical frequency $\omega_{c}^{\prime}=13 \times 10^{39} \mathrm{t}^{-1}$ (where $\mathrm{t}$ represents an arbitrary time unit.)

$$
\omega^{\prime 2}=\left|v\left(x_{H, \omega^{\prime}}\right)\right|^{2} k_{P}^{2}\left(\frac{\left|v^{2}\left(x_{H, \omega^{\prime}}\right)\right|^{2}}{c^{2}}-1\right) .
$$

Given a concrete profile, $x_{H, \omega^{\prime}}$ can then be obtained and $\kappa_{\omega^{\prime}}$ calculated explicitly.

For the profiles of the first type, see Fig. 1, the result is trivial. Indeed, $|\bar{v}(x)|$ increases linearly between the horizon corresponding to $\omega^{\prime}=0$ and the one for $\omega_{c}^{\prime}$, so we obtain a constant $\kappa_{\omega^{\prime}}=\kappa_{0}$ for all $\omega^{\prime}<\omega_{c}^{\prime}$.

For a Schwarzschild profile as in Fig. 2, on the other hand,

$$
\bar{v}(x)=-c \sqrt{\frac{c^{2} / 2 \kappa_{0}}{x+c^{2} / 2 \kappa_{0}}},
$$

so we obtain

$$
\begin{aligned}
\kappa_{\omega^{\prime}} & \equiv c\left|\frac{d \bar{v}}{d x}\right|_{x=x_{H, \omega^{\prime}}}=\kappa_{0}\left(\frac{\left|\bar{v}_{H}\right|}{c}\right)^{3 / 2} \\
& =\kappa_{0} \frac{1}{2 \sqrt{2}}\left(1+\sqrt{1+4 \frac{\omega^{\prime 2}}{c^{2} k_{P}^{2}}}\right)^{3 / 2}
\end{aligned}
$$

Note that $\kappa_{\omega^{\prime}}$ remains nearly constant until frequencies of the order of magnitude of the Planck scale are reached, and then starts to increase rapidly, see Fig. 4. As we will see graphically in the next section, this can have an important qualitative influence on the radiation spectrum.

We now have all the tools necessary to compute and plot (4.24) for different parameters of the profiles described in Sec. II.

\section{GRAPHICAL RESULTS AND DISCUSSION}

We have calculated Eq. (4.24) numerically using the Gauss-Chebyshev quadrature method. The results are plot- 
ted in Figs. 5-7 and perfectly illustrate the three important factors that we deduced theoretically in Sec. IV E. Note that in all the figures we have plotted $E \equiv \omega^{3} \times N$ against $\omega$ to make visual comparison with the usual thermal spectra in $3+1$ dimensions easier.

Figure 5 shows the influence of the critical frequency $\omega_{c}^{\prime}$ for a profile with constant $\kappa_{\omega^{\prime}}$, as in Fig. 1, and $u_{l}=\bar{u}_{I, \omega_{c}^{\prime}}$ (i.e., immediately after the horizons have formed for all $\left.\omega^{\prime}<\omega_{c}^{\prime}\right)$. The constant $\kappa_{\omega^{\prime}}$ guarantees that the form of the thermal spectrum is preserved. However, the intensity of the radiation decreases with decreasing critical frequency. Actually, only for extremely high critical frequencies is the original Hawking spectrum recovered. For a critical frequency still well above the Planck scale, the decrease can be significant. For example, for $\omega_{c}^{\prime}=10^{61} c k_{P}$, the peak intensity decreases by nearly $30 \%$, while for $\omega_{c}^{\prime}=c k_{P}$, the decrease is approximately $40 \%$. At the other end, note that extremely low critical frequencies still leave a significant amount of radiation. For example, for $\omega_{c}^{\prime}=10^{-139} c k_{P}$, still $20 \%$ of the original peak radiation is obtained. So Hawking radiation receives significant contributions from an extremely wide range of frequencies.

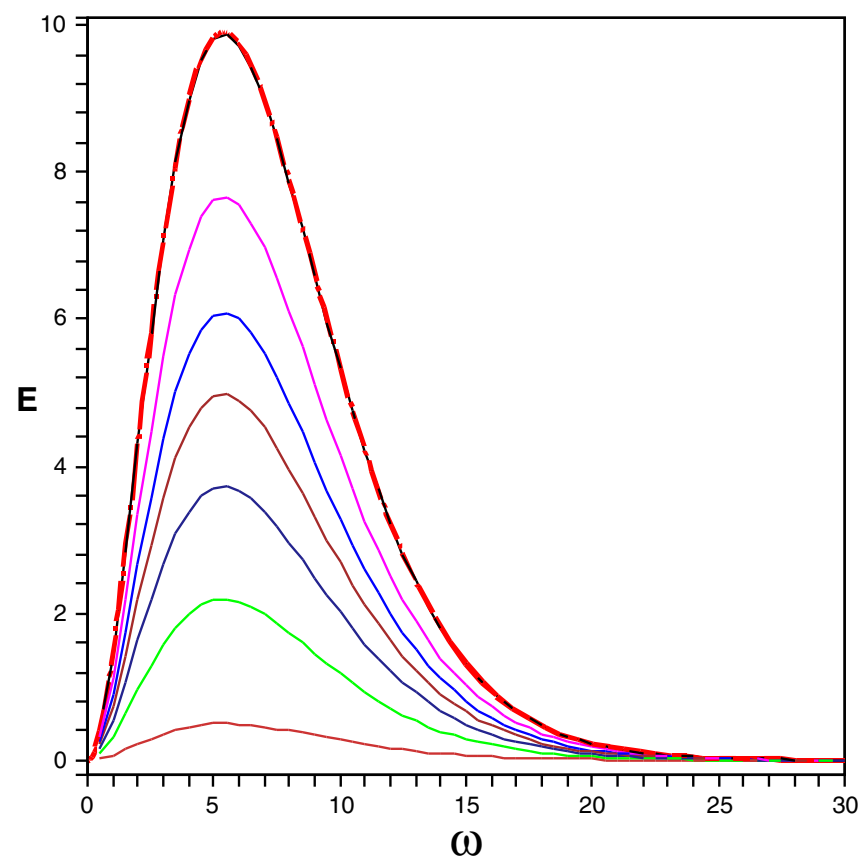

FIG. 5 (color online). Influence of the critical frequency on the radiation spectrum for a black hole with velocity profile such that the surface gravity is constant, as in Fig. 1, and different values of the critical frequency. We have chosen $c=1, u_{l}=u_{I}=0$, $U_{H}=A=1 \mathrm{t}, \kappa_{0}=12 \mathrm{t}^{-1}$ and the Planck scale $k_{P}=10^{39} \mathrm{t}^{-1}$ (where $\mathrm{t}$ represents an arbitrary time unit), which amounts to considering a solar-mass black hole. From top to bottom we have plotted $\omega_{c}^{\prime}=10^{3000}, 10^{100}, 10^{39}, 1,10^{-39}, 10^{-100}, 10^{-300}$. Note that the standard Hawking spectrum coincides perfectly with the upper curve, which effectively corresponds to the absence of a critical frequency.

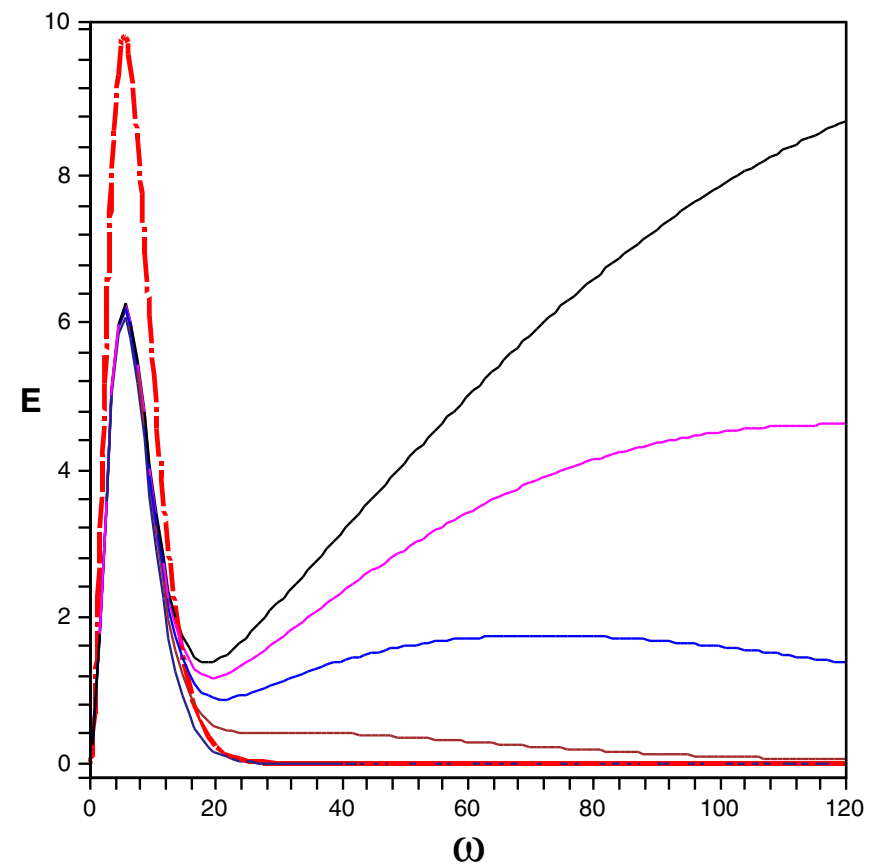

FIG. 6 (color online). Influence of a frequency-dependent surface gravity $\kappa_{\omega^{\prime}}$ on the radiation spectrum for a black hole with Schwarzschild-type velocity profile (surface gravity increases with frequency), as in Fig. 2, and different values of the critical frequency around the Planck scale $k_{P}: \omega_{c}^{\prime} / c k_{P}=13,10,7,4$, 0.1 (from top to bottom). The standard Hawking spectrum is depicted in dashed-dotted line for comparison. Numerical values of $\kappa_{0}, k_{P}$, etc. as in Fig. 5.

Figure 6 shows the radiation spectrum for a Schwarzschild-like profile and hence increasing $\kappa_{\omega^{\prime}}$, as in Fig. 2, in particular, for critical frequencies $\omega_{c}^{\prime}$ close to the Planck scale. On top of the general decrease of the standard thermal part of the spectrum (approximately $40 \%$, as in the previous case) due to the finite integration boundary induced by the critical frequency, the fact that the surface gravity is now frequency dependent leads to an important qualitative change of the spectrum. The highfrequency tail of the spectrum is totally transformed. Actually, if the critical frequency is sufficiently higher than the Planck scale, the dominant source of radiation lies in the high-frequency region. Note that this effect is truly a consequence of the modification of the physics for frequencies above the Planck scale. This can be appreciated by noticing that, at $\omega_{c}^{\prime}=0.1 c k_{P}$, the whole tailmodifying effect has disappeared and the usual thermal form of the radiation spectrum is recovered (although still with the quantitative decrease described above).

Finally, Fig. 7 shows the influence of the measuring time $u_{l}$ (measured with respect to $\bar{u}_{I, \omega_{c}^{\prime}}$ ) for a profile of the second type (increasing $\kappa_{\omega^{\prime}}$ ). It is clearly seen that the radiation dies off with time, and actually dies off rather fast. For an actual solar-mass black hole and an $\omega_{c}^{\prime}$ of the order of the Planck scale, the radiation would last only a 


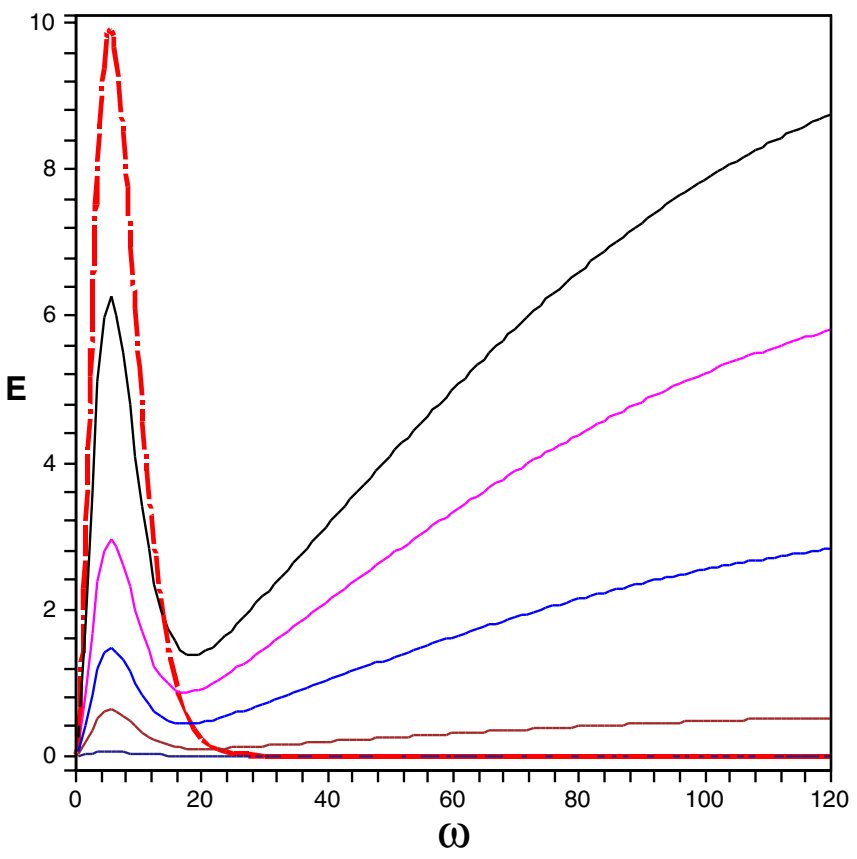

FIG. 7 (color online). Influence of the measuring time $u_{l}$ on the radiation spectrum, for a Schwarzschild-type velocity profile, see Fig. 2 and $\omega_{c}^{\prime} / c k_{P}=13$, compare with Fig. 6. Different values of $u_{l}$ (from top to bottom): $u_{l}=0,22,35,50,500 \mathrm{t}$ with $\mathrm{t}$ denoting an arbitrary time unit. The standard Hawking spectrum is depicted in the dashed-dotted line for comparison. Numerical values of $\kappa_{0}, k_{P}$ etc. as in Fig. 5.

few tens of milliseconds. Note that this effect is a corollary of the existence of a critical frequency $\omega_{c}^{\prime}$, since in its absence, the integration boundaries in (4.24) would be infinite, and so the integral would be insensitive to a change $u \rightarrow u+\Delta u$.

The combined effect of Figs. 6 and 7 leads to the following qualitative picture for the further collapse towards a singularity once the initial or classical horizon has formed. As the interior gradually uncovers a larger portion of the Schwarzschild geometry, two competing processes will take place. On the one hand, the spectrum acquires ever larger contributions associated with higher and higher temperatures. On the other hand, the overall magnitude of the spectrum is damped with time. The question of which process dominates would depend on the fine details of the dynamics of the collapse, and might be further complicated by backreaction effects, which we have not considered in our analysis.

\section{SUMMARY AND CONCLUSIONS}

We have discussed the Hawking radiation for a collapsing configuration with superluminal dispersion relations. Modifications of the dispersion relation cause the horizon, and various associated quantities such as the surface gravity $\kappa$, to become frequency dependent. In particular, a critical frequency $\omega_{c}^{\prime}$ naturally appears such that frequen- cies higher than $\omega_{c}^{\prime}$ do not experience a horizon at all. More generally, it also means that the standard geometric concepts traditionally used to study black holes must be handled with care. Nevertheless, through a detailed analysis, we have seen that the equations related to the late-time radiation can be adapted quite straightforwardly from standard (relativistic) to superluminal dispersion relations.

We analytically derived an approximate equation for the particle production at late times (4.24) with superluminal modifications of the dispersion relations. This equation clearly showed that important modifications in the latetime radiation should be expected, first, due to the existence of the critical frequency $\omega_{c}^{\prime}$ and the finite upper boundary it induces in the integration, and second, due to the frequency dependence of $\kappa_{\omega^{\prime}}$. We integrated (4.24) numerically, and plotted the resulting spectrum, thereby confirming these expectations.

We have seen that the standard Hawking spectrum is recovered only in a very particular case: in the limit when the critical frequency goes to infinity (i.e., when the profile for the velocity $|v|$ goes to a singularity) and moreover the surface gravity $\kappa_{\omega^{\prime}}$ is constant (linear velocity profiles of the type of Fig. 1). For lower critical frequencies, as long as $\kappa$ remains constant, the thermal form of the Hawking spectrum is maintained, but the intensity decreases rapidly with decreasing $\omega_{c}^{\prime}$. A non-negligible radiation persists, however, even for extremely low critical frequencies. This clearly establishes the first point, namely, the importance of the critical frequency $\omega_{c}^{\prime}$, which illustrates the statement made in the introduction that with superluminal dispersion relations, the interior of the black hole is probed, which can significantly affect the Hawking process.

For a Schwarzschild-like profile, the velocity profile generally leads to a frequency-dependent surface gravity $\kappa_{\omega^{\prime}}$, increasing with the frequency $\omega^{\prime}$. This means that the standard Hawking result cannot be recovered for a Schwarzschild black hole with superluminal dispersion relations, even when the velocity profile has a singularity. Actually, when the velocity profile has a limiting value, the same quantitative decrease of the Hawking part of the spectrum as before shows up. Moreover, if the critical frequency $\omega_{c}^{\prime}$ is above the Planck scale, a drastic qualitative change of the radiation spectrum takes place, and for sufficiently high $\omega_{c}^{\prime}$ (roughly a few times the Planck scale) the high-frequency part of the spectrum even becomes dominant. This shows the importance of the surface gravity, which again illustrates the role played by the interior of the black hole.

Finally, we have also seen that, as a corollary of the existence of a critical frequency and of the finite upper boundary induced by it in the thermal response function, the radiation spectrum dies off as time advances.

A few observations might be useful to connect our work with existing results on Hawking radiation and its sensitivity to modified dispersion relations. The most general 
observation is that the "robustness" of Hawking radiation, which is often considered to be a well-established result, actually depends crucially on a series of assumptions. These assumptions might be reasonable in the case of subluminal dispersion relations. But for the case of superluminal dispersion relations, as we have shown explicitly, the assumptions needed to reproduce the standard Hawking result depend on the physics inside the (zerofrequency) horizon, and moreover in a way which is not compatible with the Schwarzschild geometry. In particular, in [20], it was shown for a stationary scenario that Hawking radiation is robust with respect to superluminal modifications of the dispersion relations, provided that positive free-fall frequency modes were in their ground state just before crossing the horizon. However, it was also admitted that it is not clear whether this is the physically correct quantum state condition. In [21], three explicit assumptions were given for the previous condition to hold: freely falling frame, ground state, and adiabatic evolution. In the same article, it is similarly pointed out that these assumptions might fail for a superluminal dispersion relation, since (as we also mentioned in the introduction) for highfrequency modes this implies that one makes an assumption about the physics at the singularity. Rather than imposing any conditions on the state near the horizon, and hence ultimately at the singularity, we have fixed the initial geometry to be Minkowskian and the quantum field to be in the natural associated vacuum state, and evolved this into a black hole configuration.

Since our results are seemingly in contradiction with the ones obtained in $[20,21]$, it is worth examining in which sense the conditions stated by those authors are violated in our approach. If one considers a collapse scenario, for example, of a BEC, in a laboratory setting, then it is quite natural to assume that the "freely falling frame" condition will be violated. Indeed, the superluminal modification is in this case associated with the existence of a privileged external reference frame: the black hole rest frame or lab frame (as we noted in Sec. IVA), and not the freely falling frame, as assumed in [21]. Note that this violation of the free-fall frame condition automatically implies a violation of the ground state condition in the sense in which this condition is formulated in [21], namely, as the ground state "with respect to the freely falling frame." Indeed, our results suggest the following picture. The low-energy modes experience the classical geometry and are therefore dragged along in the free-falling frame and so at the horizon they occupy the vacuum state associated with this free-falling frame, namely, the Unruh vacuum. Hence they contribute to the black hole radiation in the traditional Hawking way. However, the ultrahighfrequency modes (above the critical frequency) do not see the horizon. Hence they do not couple to the classical geometry of the collapse, but rather remain connected to the external or laboratory frame, and therefore pass through the black hole (nearly) undisturbed. The ground state of these high-frequency modes is then not the vacuum associated with the freely falling frame, but the Boulware vacuum associated with the original Minkowski geometry, or in other words, with the stationary reference frame of the lab. So these frequencies above the critical frequency do not contribute to the thermal output spectrum. The overall radiation is a convolution of the contributions from all the different initial frequencies, where the surface gravity for each frequency can be interpreted as leading to an effective weighting factor. Depending on the internal physics of the black hole, this leads to either a spectrum of the traditional Hawking form but with a reduced intensity (in the case of a constant surface gravity), or a modified spectrum where the high-frequency contributions dominate (as for a Schwarzschild profile, provided that the critical frequency lies sufficiently above the Planck scale).

In any case, what our analysis shows with respect to the situation treated in $[20,21]$ is the following. The robustness of Hawking radiation discussed by those authors is the robustness with respect to a superluminal modification under the precise conditions occurring in the standard case, in which all modes occupy the ground state associated with the free-falling frame just before crossing the horizon. This assumption implies that all modes, regardless of their frequency, occupy the Unruh vacuum. Our analysis shows precisely that the superluminality can lead to a spontaneous breaking of these conditions. The natural evolution of a collapsing configuration is such that the frequencies above the critical frequency $\omega_{c}^{\prime}$ are not in the Unruh vacuum associated with the freely falling frame, but remain in the Boulware vacuum associated with the initial asymptotic Minkowski condition. It should then come as no surprise that this can indeed have an important impact on the resulting radiation spectrum.

We should mention that in a recent paper by Schützhold and Unruh [22] based on an alternative approximation method, a new potential problem with superluminal dispersion relations is highlighted. This "ultraviolet catastrophe" is associated with a higher-than-linear growth of the group velocity at large wave numbers, and should therefore not occur for the dispersion relation used in our work. However, we have found that a Schwarzschild interior geometry could give place to the same sort of phenomenology.

A final observation concerns the recent article [33], in which the authors numerically simulate the formation of an acoustic horizon in a BEC and analyze the creation of correlated pairs of phonons through the so-called truncated Wigner method. Our findings for the case of a constant surface gravity seem to be in qualitative disagreement with the discussion presented in [33], since the authors of this paper assert having observed a stationary Hawking flux, while our analysis concludes that the radiation should quickly fade off. The source of this apparent discrepancy 
might reside in the fact that the correlation function which they study is normalized, and therefore probes the form of the spectrum (which we also find stationary with time), but does not provide details about the net amount of particle production.

\section{ACKNOWLEDGMENTS}

We kindly thank Stefano Liberati and Matt Visser for enlightening comments and discussions, and José Luis
Jaramillo for very useful suggestions on the numerical aspects of this work. G. J. would also like to express his gratitude to Fernando Barbero, Daniel Gómez Vergel, and Lorenzo Sindoni for useful discussions, and SISSA (Trieste) for hospitality. Financial support was provided by the Spanish MEC through the projects FIS2005-05736C03-01, FIS2005-05736-C03-02, and FIS2006-26387-E.
[1] T. Jacobson, S. Liberati, and D. Mattingly, Ann. Phys. (N.Y.) 321, 150 (2006).

[2] N.E. Mavromatos, Proc. Sci., QG-PH (2007) 027 [arXiv:0708.2250].

[3] R. Gambini and J. Pullin, Phys. Rev. D 59, 124021 (1999).

[4] A. Ashtekar, arXiv:0705.2222.

[5] C. Barceló, S. Liberati, and M. Visser, Living Rev. Relativity 8, 12 (2005), http://www.livingreviews.org/lrr2005-12.

[6] G.E. Volovik, The Universe in a Helium Droplet (Clarendon Press, Oxford, England, 2003).

[7] F. R. Klinkhamer and G.E. Volovik, Pis'ma Zh. Eksp. Teor. Fiz. 81, 683 (2005); [JETP Lett. 81, 551 (2005)].

[8] D. Mattingly, Living Rev. Relativity 8, 5 (2005).

[9] J. Magueijo and L. Smolin, Phys. Rev. Lett. 88, 190403 (2002).

[10] S. W. Hawking, Nature (London) 248, 30 (1974).

[11] S. W. Hawking, Commun. Math. Phys. 43, 199 (1975).

[12] I. Agullo, J. Navarro-Salas, G. J. Olmo, and L. Parker, Phys. Rev. D 76, 044018 (2007).

[13] W. G. Unruh, Phys. Rev. Lett. 46, 1351 (1981).

[14] T. Jacobson, Phys. Rev. D 44, 1731 (1991).

[15] T. Jacobson, Phys. Rev. D 48, 728 (1993).

[16] W. G. Unruh, Phys. Rev. D 51, 2827 (1995).

[17] R. Brout, S. Massar, R. Parentani, and P. Spindel, Phys. Rev. D 52, 4559 (1995).

[18] S. Corley and T. Jacobson, Phys. Rev. D 54, 1568 (1996).

[19] S. Corley, Phys. Rev. D 55, 6155 (1997).

[20] S. Corley, Phys. Rev. D 57, 6280 (1998).

[21] W. G. Unruh and R. Schützhold, Phys. Rev. D 71, 024028 (2005).

[22] R. Schützhold and W. G. Unruh, Phys. Rev. D 78, 041504 (2008).
[23] M. Visser, Int. J. Mod. Phys. D 12, 649 (2003).

[24] C. Barceló, A. Cano, L. J. Garay, and G. Jannes, Phys. Rev. D 74, 024008 (2006).

[25] S. Corley and T. Jacobson, Phys. Rev. D 59, 124011 (1999).

[26] L. J. Garay, J. R. Anglin, J. I. Cirac, and P. Zoller, Phys. Rev. Lett. 85, 4643 (2000).

[27] L. J. Garay, J. R. Anglin, J. I. Cirac, and P. Zoller, Phys. Rev. A 63, 023611 (2001).

[28] C. Barceló, S. Liberati, and M. Visser, Classical Quantum Gravity 18, 1137 (2001).

[29] C. Barceló, S. Liberati, and M. Visser, Int. J. Mod. Phys. A 18, 3735 (2003).

[30] R. Schützhold, Phys. Rev. Lett. 97, 190405 (2006).

[31] S. Wüster and C. M. Savage, Phys. Rev. A 76, 013608 (2007).

[32] R. Balbinot, A. Fabbri, and S. Fagnocchi, Phys. Rev. A 78, 021603 (2008).

[33] I. Carusotto, S. Fagnocchi, A. Recati, R. Balbinot, and A. Fabbri, New J. Phys. 10, 103001 (2008).

[34] S. Wüster, Phys. Rev. A 78, 021601(R) (2008).

[35] M. Visser, Classical Quantum Gravity 15, 1767 (1998).

[36] A. Fabbri and J. Navarro-Salas, Modeling Black Hole Evaporation (Imperial College Press, London, 2005).

[37] M. Visser and S. Weinfurtner, Proc. Sci. QG-PH (2007) 042 [arXiv:0712.0427].

[38] M. Visser, arXiv:0712.0810.

[39] C. Barceló, S. Liberati, S. Sonego, and M. Visser, Classical Quantum Gravity 23, 5341 (2006).

[40] C. Barceló, S. Liberati, S. Sonego, and M. Visser, Phys. Rev. Lett. 97, 171301 (2006). 